\title{
Majorization for certain classes of analytic functions defined by convolution structure
}

\author{
Ekram Elsayed Ali
}

\begin{abstract}
In this paper, we investigate majorization properties for certain classes of analytic functions defined by convolution structure. Also we point out some new and known consequences of our main result.
\end{abstract}

Mathematics Subject Classification (2010): 30C45.

Keywords: Analytic functions, starlike function, Hadamard product, majorization.

\section{Introduction}

Let $f(z)$ and $g(z)$ be analytic in the open unit disc $U=\{z \in \mathbb{C}:|z|<1\}$.

For analytic function $f(z)$ and $g(z)$ in $U$, we say that $f(z)$ is majorized by $g(z)$ in $U$ (see [10]) and write

$$
f(z)<<g(z) \quad(z \in U),
$$

if there exists a function $\varphi(z)$, analytic in $U$ such that

$$
|\varphi(z)| \leq 1 \quad \text { and } \quad f(z)=\varphi(z) g(z) \quad(z \in U) .
$$

It may be noted that (1.1) is closely related to the concept of quasi-subordination between analytic functions.

If $f(z)$ and $g(z)$ are analytic functions in $U$, we say that $f(z)$ is subordinate to $g(z)$, written symbolically as $f(z) \prec g(z)$ if there exists a Schwarz function $w$, which (by definition) is analytic in $U$ with $w(0)=0$ and $|w(z)|<1$ for all $z \in U$, such that $f(z)=g(w(z)), z \in U$. Furthermore, if the function $g(z)$ is univalent in $U$, then we have the following equivalence, (see $[11, \mathrm{p} .4]$ ):

$$
f(z) \prec g(z) \Leftrightarrow f(0)=g(0) \text { and } f(U) \subset g(U) .
$$

Let $A(p)$ denote the class of functions $f(z)$ of the form:

$$
f(z)=z^{p}+\sum_{k=p+1}^{\infty} a_{k} z^{k}, \quad(p \in \mathbb{N}=\{1,2, \ldots \ldots\})
$$


which are analytic and $p$-valent in the open unit disc. We note that $A(1)=A$. Let $g(z) \in A(p)$, be given by

$$
g(z)=z^{p}+\sum_{k=p+1}^{\infty} b_{k} z^{k}
$$

the Hadamard product (or convolution) of $f(z)$ and $g(z)$ is given by

$$
(f * g)(z)=z^{p}+\sum_{k=p+1}^{\infty} a_{k} b_{k} z^{k}=(g * f)(z) .
$$

For $\lambda, \ell \geqslant 0, p \in \mathbb{N}, m \in \mathbb{N}_{0}=\mathbb{N} \cup\{0\}$ and $f(z), g(z) \in A(p)$, A. O. Mostafa, [12] defined the linear operator $D_{\lambda, \ell, p}^{m}(f * g)$ as follows:

$$
D_{p, \ell, \lambda}^{m}(f * g)=z^{p}+\sum_{k=p+1}^{\infty}\left[\frac{p+\ell+\lambda(k-p)}{p+\ell}\right]^{m} a_{k} b_{k} z^{k} .
$$

From (1.5), it is easy to verify that ( see [12]),

$$
\lambda z\left(D_{\lambda, \ell, p}^{m}(f * g)(z)\right)^{\prime}=(\ell+p) D_{\lambda, \ell, p}^{m+1}(f * g)(z)-[p(1-\lambda)+\ell] D_{\lambda, \ell, p}^{m}(f * g)(z) .
$$

We note that:

(i) For $b_{k}=1$ or $g(z)=\frac{z^{p}}{1-z}$ we have $D_{\lambda, \ell, p}^{m} f(z)=I_{p}^{m}(\lambda, \ell) f(z)$, where the operator $I_{p}^{m}(\lambda, \ell)$ was introduced and studied by Cătaş [4], which contains intern the operators $D_{p}^{m}$, (see [2] and [8]) and $D_{\lambda}^{m}$ (see [1]).

(ii) For $b_{k}=\frac{\left(\alpha_{1}\right)_{k-p} \ldots\left(\alpha_{q}\right)_{k-p}}{\left(\beta_{1}\right)_{k=p} \ldots\left(\beta_{s}\right)_{k-p}(1)_{k-p}}$, the operator

$$
D_{\lambda, \ell, p}^{m}(f * g)(z)=I_{p, q, r, \lambda}^{m, \ell}\left(\alpha_{1}, \beta_{1}\right) f(z),
$$

where the operator $I_{p, q, r, \lambda}^{m, \ell}\left(\alpha_{1}, \beta_{1}\right) f(z)$ was introduced and studied by El-Ashwah and Aouf [6], $\alpha_{1}, \alpha_{2}, \ldots, \alpha_{q}$ and $\beta_{1}, \beta_{2}, \ldots, \beta_{s}$ are real or complex number $\left(\beta_{j} \in \mathbb{C} \backslash \mathbb{Z}_{0}^{-}=\right.$ $\{0,-1,-2, \ldots\} ; j=1, \ldots, s ;)\left(q \leq s+1 ; q, s \in \mathbb{N}_{0}, p \in \mathbb{N} ; z \in U\right)$ and

$$
(\theta)_{\nu}=\frac{\Gamma(\theta+\nu)}{\Gamma(\theta)}= \begin{cases}1 & \left(\nu=0 ; \theta \in \mathbb{C}^{*}=\mathbb{C} \backslash\{0\}\right), \\ \theta(\theta-1) \ldots(\theta+\nu-1) & (\nu \in \mathbb{N} ; \theta \in \mathbb{C}) .\end{cases}
$$

Also, for many special operators of the operator $I_{p, q, r, \lambda}^{m, \ell}\left(\alpha_{1}, \beta_{1}\right) f(z)$ (see [6]).

(iii) For $m=0, b_{k}=\frac{\left(\alpha_{1}\right)_{k-p} \ldots\left(\alpha_{q}\right)_{k-p}}{\left(\beta_{1}\right)_{k=p} \ldots\left(\beta_{s}\right)_{k-p}(1)_{k-p}}$, the operator

$$
D_{\lambda, \ell, p}^{m}(f * g)(z)=S_{p, q, s}^{j}\left(\gamma ; \alpha_{1}\right) f(z),
$$

where the operator $S_{p, q, s}^{j}\left(\gamma ; \alpha_{1}\right) f(z)$, was introduced and studied by El-Ashwah [5].

(iv) For $m=0$ and $b_{k}=\frac{\Gamma(p+\alpha+\beta) \Gamma(k+\beta)}{\Gamma(p+\beta) \Gamma(k+\alpha+\beta)}$, the operator $D_{p, \ell, \lambda}^{m}(f * g)(z)=Q_{p, \beta}^{\alpha}(f)$ $(\alpha \geq 0, \beta>-1, p \in \mathbb{N})$, where the operator $Q_{p, \beta}^{\alpha}$ was introduced by Liu and Owa [9].

For $h(z)$ given by

$$
h(z)=z^{p}+\sum_{k=p+1}^{\infty} c_{k} z^{k}
$$


A function $f(z) \in A(p)$ is said to be in the class $S_{\lambda, \ell, p}^{m, j}(\gamma)$ of $p$-valent functions of complex order $\gamma \neq 0$ in $U$, if and only if

$$
\begin{aligned}
& \operatorname{Re}\left\{1+\frac{1}{\gamma}\left(\frac{z\left(D_{\lambda, \ell, p}^{m}(f * h)(z)\right)^{(j+1)}}{\left(D_{\lambda, \ell, p}^{m}(f * h)(z)\right)^{(j)}}-p+j\right)\right\}>0 \\
& \left(p \in \mathbb{N} ; j \in \mathbb{N}_{0}=\mathbb{N} \cup\{0\} ; \ell, \lambda \geq 0 ; \gamma \in \mathbb{C}^{*} ; z \in U\right) .
\end{aligned}
$$

Clearly, we have the following relationships:
(i) $S_{\lambda, \ell, 1}^{0,0}(\gamma)=S(\gamma)\left(\gamma \in \mathbb{C}^{*}\right)$,
(ii) $S_{\lambda, \ell, 1}^{0,1}(\gamma)=\kappa(\gamma)\left(\gamma \in \mathbb{C}^{*}\right)$,
(iii) $S_{\lambda, \ell, 1}^{0,0}(1-\alpha)=S^{*}(\alpha)(0 \leq \alpha<1)$.

The classes $S(\gamma)$ and $\kappa(\gamma)$ are classes of starlike and convex functions of complex order $\gamma \neq 0$ in $U$ which were studied by Nasr and Aouf [13] and $S^{*}(\alpha)$ is the class of starlike functions of order $\alpha$ in $U$.

Also, for $m=0$ the operator $S_{p}^{j}(h ; \gamma)$ was introduced and studied by El-Ashwah and Aouf [7].

Definition 1.1. Let $-1 \leq B<A \leq 1, p \in \mathbb{N} ; j \in \mathbb{N}_{0}, \gamma \in \mathbb{C}^{*}$,

$$
|\gamma(A-B)+(p-j) B|<(p-j), f \in A(p) .
$$

Then $f \in S_{\lambda, \ell, p}^{m, j}(\gamma ; A, B)$, the class of $p$-valent functions of complex order $\gamma$ in $U$ if and only if

$$
\left\{1+\frac{1}{\gamma}\left(\frac{z\left(D_{\lambda, \ell, p}^{m}(f * h)(z)\right)^{(j+1)}}{\left(D_{\lambda, \ell, p}^{m}(f * h)(z)\right)^{(j)}}-p+j\right)\right\} \prec \frac{1+A z}{1+B z} .
$$

A majorization problem for the subclasses of analytic function has recently been investigated by Altintas et al. [3] and MacGregor [11]. In this paper we investigate majorization problem for the class $S_{\lambda, \ell, p}^{m, j}(\gamma ; A, B)$ and some related subclasses.

\section{Main results}

Unless otherwise mentioned we shall assume throughout the paper that, $-1 \leq$ $B<A \leq 1, \gamma \in \mathbb{C}^{*}, \ell, \lambda \geq 0, p \in \mathbb{N}$ and $m, j \in \mathbb{N}_{0}$.

Theorem 2.1. Let the function $f \in A(p)$ and suppose that $g \in S_{\lambda, \ell, p}^{m, j}(\gamma ; A, B)$. If $\left(D_{\lambda, \ell, p}^{m}(f * h)(z)\right)^{(j)}$ is majorized by $\left(D_{\lambda, \ell, p}^{m}(g * h)(z)\right)^{(j)}$ in $U$, then

$$
\left|\left(D_{\lambda, \ell, p}^{m+1}(f * h)(z)\right)^{(j)}\right| \leq\left|\left(D_{\lambda, \ell, p}^{m+1}(g * h)(z)\right)^{(j)}\right| \quad\left(|z|<r_{1}\right),
$$

where $r_{1}=r_{1}(p, \gamma, \lambda, \ell, A, B)$ is the smallest positive root of the equation

$$
\begin{gathered}
|\gamma \lambda(A-B)+(p+\ell) B| r^{3}-[2 \lambda|B|+(p+\ell)] r^{2}- \\
{[|\gamma \lambda(A-B)+(p+\ell) B|+2 \lambda] r+(p+\ell)=0 .}
\end{gathered}
$$

Proof. Since $(g * h)(z) \in S_{\lambda, \ell, p}^{m, j}(\gamma ; A, B)$, we find from (1.8) that

$$
1+\frac{1}{\gamma}\left(\frac{z\left(D_{\lambda, \ell, p}^{m}(g * h)(z)\right)^{(j+1)}}{\left(D_{\lambda, \ell, p}^{m}(g * h)(z)\right)^{(j)}}-p+j\right)=\frac{1+A w(z)}{1+B w(z)},
$$


where $w$ is analytic in $U$ with $w(0)=0$ and $|w(z)|<1(z \in U)$. From (2.3), we have

$$
\frac{z\left(D_{\lambda, \ell, p}^{m}(g * h)(z)\right)^{(j+1)}}{\left(D_{\lambda, \ell, p}^{m}(g * h)(z)\right)^{(j)}}=\frac{(p-j)+[\gamma(A-B)+(p-j) B] w(z)}{1+B w(z)} .
$$

In view of

$$
\begin{gathered}
\lambda z\left(D_{\lambda, \ell, p}^{m}(f * g)(z)\right)^{(j+1)}=(p+\ell)\left(D_{\lambda, \ell, p}^{m+1}(f * g)(z)\right)^{(j)} \\
-[p(1-\lambda)+\lambda j+\ell]\left(D_{\lambda, \ell, p}^{m}(f * g)(z)\right)^{(j)} \\
0 \leq j \leq p ; p \in \mathbb{N}, \lambda>0 ; z \in U
\end{gathered}
$$

(2.4) immediately yields the following inequality:

$$
\left|\left(D_{\lambda, \ell, p}^{m}(g * h)(z)\right)^{(j)}\right| \leq \frac{(p+\ell)(1+|B||z|)}{(p+\ell)-|\gamma \lambda(A-B)+(p+\ell) B||z|}\left|\left(D_{\lambda, \ell, p}^{m+1}(g * h)(z)\right)^{(j)}\right| .
$$

Next, since $\left(D_{\lambda, \ell, p}^{m}(f * h)(z)\right)^{(j)}$ is majorized by $\left(D_{\lambda, \ell, p}^{m}(g * h)(z)\right)^{(j)}$ in $U$, from $(1.2)$, we have

$$
\left(D_{\lambda, \ell, p}^{m}(f * h)(z)\right)^{(j)}=\varphi(z)\left(D_{\lambda, \ell, p}^{m}(g * h)(z)\right)^{(j)} .
$$

Differentiating (2.7) with respect to $z$, we have

$$
z\left(D_{\lambda, \ell, p}^{m}(f * h)(z)\right)^{(j+1)}=z \varphi^{\prime}(z)\left(D_{\lambda, \ell, p}^{m}(g * h)(z)\right)^{(j)}+z \varphi(z)\left(D_{\lambda, \ell, p}^{m}(g * h)(z)\right)^{(j+1)} \text {. }
$$

From (2.5) and (2.8), we have

$$
\left(D_{\lambda, \ell, p}^{m+1}(f * h)(z)\right)^{(j)}=\frac{\lambda z}{p+\ell} \varphi^{\prime}(z)\left(D_{\lambda, \ell, p}^{m}(g * h)(z)\right)^{(j)}+\varphi(z)\left(D_{\lambda, \ell, p}^{m+1}(g * h)(z)\right)^{(j)} .
$$

Thus, by noting that $\varphi(z)$ satisfies the inequality (see [14]),

$$
\left|\varphi^{\prime}(z)\right| \leq \frac{1-|\varphi(z)|^{2}}{1-|z|^{2}} \quad(z \in U)
$$

we see that

$$
\leq\left(|\varphi(z)|+\frac{1-|\varphi(z)|^{2}}{1-|z|^{2}} \cdot \frac{\left|\left(D_{\lambda, \ell, p}^{m+1}(f * h)(z)\right)^{(j)}\right|}{(p+\ell)-|\gamma \lambda(A-B)+(p+\ell) B||z|}\right)\left|\left(D_{\lambda, \ell, p}^{m+1}(g * h)(z)\right)^{(j)}\right|
$$

which upon setting

$$
|z|=r \quad \text { and } \quad|\varphi(z)|=\rho \quad(0 \leq \rho \leq 1)
$$

leads us to the inequality

$$
\begin{aligned}
& \leq\left|\left(D_{\lambda, \ell, p}^{m+1}(f * h)(z)\right)^{(j)}\right| \\
& \leq \frac{\Theta(\rho)}{\left(1-r^{2}\right)((p+\ell)-|\gamma \lambda(A-B)+(p+\ell) B| r)}\left|\left(D_{\lambda, \ell, p}^{m+1}(g * h)(z)\right)^{(j)}\right|,
\end{aligned}
$$

where

$$
\begin{gathered}
\Theta(\rho)=-r \lambda(1+|B| r) \rho^{2}+\left(1-r^{2}\right)[(p+\ell)-|\gamma \lambda(A-B)+(p+\ell) B| r] \rho \\
+r \lambda(1+|B| r)
\end{gathered}
$$


takes its maximum value at $\rho=1$, with $r_{1}=r_{1}(p, \gamma, \lambda, \ell, A, B)$, where $r_{1}(p, \gamma, \lambda, \ell, A, B)$ is the smallest positive root of $(2.2)$. Therefore the function $\Phi(\rho)$ defined by

$$
\begin{gathered}
\Phi(\rho)=-\sigma \lambda(1+|B| \sigma) \rho^{2}+\left(1-\sigma^{2}\right)[(p+\ell)-|\gamma \lambda(A-B)+(p+\ell) B| \sigma] \rho \\
+\sigma \lambda(1+|B| \sigma)
\end{gathered}
$$

is an increasing function on the interval $0 \leq \rho \leq 1$, so that

$$
\begin{gathered}
\Phi(\rho) \leq \Phi(1)=\left(1-\sigma^{2}\right)[(p+\ell)-|\gamma(A-B)+(p+\ell) B| \sigma] \\
\left(0 \leq \rho \leq 1 ; 0 \leq \sigma \leq r_{0}(p, \gamma, j, A, B)\right) .
\end{gathered}
$$

Hence upon setting $\rho=1$ in (2.12), we conclude that (2.1) holds true for $|z| \leq$ $r_{1}=r_{1}(p, \gamma, \lambda, \ell, A, B)$, where $r_{1}(p, \gamma, \lambda, \ell, A, B)$, is the smallest positive root of $(2.2)$. This completes the proof of Theorem 1 .

Putting $A=1$ and $B=-1$ in Theorem 1, we obtain the following result.

Corollary 2.2. Let the function $f \in A(p)$ and suppose that $g \in S_{\lambda, \ell, p}^{m, j}(\gamma)$.

If $\left(D_{\lambda, \ell, p}^{m}(f * h)(z)\right)^{(j)}$ is majorized by $\left(D_{\lambda, \ell, p}^{m}(g * h)(z)\right)^{(j)}$ in $U$, then

$$
\left|\left(D_{\lambda, \ell, p}^{m+1}(f * h)(z)\right)^{(j)}\right| \leq\left|\left(D_{\lambda, \ell, p}^{m+1}(g * h)(z)\right)^{(j)}\right| \quad\left(|z|<r_{1}\right),
$$

where $r_{1}=r_{1}(p, \gamma, \lambda, \ell)$ is given by

$$
r_{1}=r_{1}(p, \gamma, \lambda, \ell)=\frac{k-\sqrt{k^{2}-4(p+\ell)|2 \gamma \lambda-(p+\ell)|}}{2|2 \gamma \lambda-(p+\ell)|},
$$

where $k=2 \lambda+(p+\ell))+\mid 2 \gamma \lambda-(p+\ell)) \mid$.

Putting $A=1, B=-1$ and $p=j=1$ in Theorem 1, we obtain the following result.

Corollary 2.3. Let the function $f \in A$ and suppose that $g \in S_{\lambda, \ell}^{m, 0}(\gamma)$.

If $\left(D_{\lambda, \ell}^{m}(f * h)(z)\right)$ is majorized by $\left(D_{\lambda, \ell}^{m}(g * h)(z)\right)$ in $U$, then

$$
\left|\left(D_{\lambda, \ell}^{m+1}(f * h)(z)\right)\right| \leq\left|\left(D_{\lambda, \ell}^{m+1}(g * h)(z)\right)\right| \quad\left(|z|<r_{2}\right),
$$

where $r_{2}=r_{2}(\gamma, \lambda, \ell)$ is given by

$$
r_{2}=r_{2}(\gamma, \lambda, \ell)=\frac{k-\sqrt{k^{2}-4(1+\ell)|2 \gamma \lambda-(1+\ell)|}}{2|2 \gamma \lambda-(1+\ell)|},
$$

where $k=2 \lambda+(1+\ell))+\mid 2 \gamma \lambda-(1+\ell)) \mid$.

Putting $A=\lambda=1, B=-1, m=\ell=0$, and $h(z)=\frac{z^{p}}{1-z}\left(\right.$ or $\left.c_{k+p}=1\right)$ in Theorem 1, we obtain the following result.

Corollary 2.4. Let the function $f \in A(p)$ and suppose that $g \in S_{p}$. If $f(z)$ is majorized by $g(z)$ in $U$, then

$$
\left|f^{\prime}(z)\right| \leq\left|g^{\prime}(z)\right| \quad\left(|z|<r_{3}\right)
$$

where $r_{3}=r_{3}(p, \gamma)$ is given by

$$
r_{3}=r_{3}(p ; \gamma)=\frac{k-\sqrt{k^{2}-4 p|2 \gamma-p|}}{2|2 \gamma-p|},
$$

where $k=2+p+|2 \gamma-p|$. 
Putting $\gamma=1$ in Corollary 3, we obtain the following result.

Corollary 2.5. Let the function $f \in A(p)$ and suppose that $g \in S_{p}(\gamma)$. If $f(z)$ is majorized by $g(z)$ in $U$, then

$$
\left|f^{\prime}(z)\right| \leq\left|g^{\prime}(z)\right| \quad\left(|z|<r_{4}\right),
$$

where $r_{4}$ is given by

$$
r_{4}=r_{4}(p)=\frac{k-\sqrt{k^{2}-4 p|2-p|}}{2|2-p|},
$$

where $k=2+p+|2-p|$

Remarks 2.6. (i) Putting $p=1$ in Corollary 3 we obtain the results obtained by Altintas et al. [3],

(ii) Putting $p=1$ in Corollary 4 we obtain the results obtained by MacGregor [10].

Acknowledgements. The author thank the referees for their valuable suggestions to improve the paper.

\section{References}

[1] Al-Oboudi, F.M., On univalent function defined by a generalized Salagean operator, Internat. J. Math. Math. Sci., 27(2004), 1429-1436.

[2] Aouf, M.K., Mostaafa, A.O., On a subclass of $n-p$-valent prestarlike functions, Comput. Math. Appl., (2008), no. 55, 851-861.

[3] Altintas, O., Ozkan, O., Srivastava, H.M., Majorization by starlike functions of complex order, Complex Var., 46(2001), 207-218.

[4] Catas, A., On certain classes of p-valent functions defined by multiplier transformations, in Proceedings of the International Symposium on Geometric Function Theory and Applications: GFTA 2007 Proceedings (Istanbul, Turkey; 20-24 August 2007) (S. Owa and Y. Polatoglu, eds.), TC Istanbul Kültür University Publications, Vol. 91, TC Istanbul Kültür University, Istanbul, Turkey, 2008, 241-250,

[5] El-Ashwah, R.M., Majorization properties for subclass of analytic p-valent functions defined by the generalized hypergeometric function, Tamsui. Oxf. J. Infor. Math. Scie., 28(2012), no. 4, 395-405.

[6] El-Ashwah, R.M., Aouf, M.K., Differential subordination and superordination for certain subclasses of p-valent functions, Math. Comput Modelling, 51(2010), 349-360.

[7] El-Ashwah, R.M., Aouf, M.K., Majorization properties for subclasses of analytic p-valent functions defined by convolution, Kyungpook Math. J., 53(2013), 615-624.

[8] Kamali, M., Orhan, H., On a subclass of certian starlike functions with negative coeffcients, Bull. Korean Math. Soc., 41(2004), no. 1, 53-71.

[9] Liu, J.L., Owa, S., Properties of certain integral operators, Internat. J. Math. Math. Sci., 3(2004), no. 1, 69-75.

[10] MacGregor, T.H., Majorization by univalent functions, Duke Math. J., 34(1967), 95-102.

[11] Miller, S.S., Mocanu, P.T., Differential Subordinations: Theory and Applications, Series on Monographs and Textbooks in Pure and Applied Mathematics, Vol. 225, Marcel Dekker Inc., New York. and Basel, 2000.

[12] Mostafa, A.O., Differential sandwich theorem for $p$-valent functions related to certain operator, Acta Univ. Apulensis, 30(2012), 221-235. 
[13] Nasr, M.A., Aouf, M.K., Starlike function of complex order, J. Natur. Sci. Math., 25(1985), no. 1, 1-12.

[14] Nehari, Z., Conformal Mapping, MacGraw-Hill Book Company, New York, Toronto and London, 1952.

Ekram Elsayed Ali

Port Said University

Faculty of Science

Department of Mathematics

Port Said 42521, Egypt

e-mail: ekram_008eg@yahoo.com 